\title{
Preliminary study of the clonal characteristics of the TCR BV subfamilies in T cells in the peripheral blood from patients with uveitis
}

\author{
H.-Y. Zou ${ }^{1}$, W.-Z. Yu ${ }^{1}$, Q. Zhang ${ }^{1}$, H.-C. Yang ${ }^{2}$, H.-Y. Huang ${ }^{3}$ and M. Jiao ${ }^{1}$ \\ ${ }^{1}$ Clinical Laboratory Diagnostics Center, \\ Urumqi General Hospital of Lanzhou Military Command Area, Urumqi, China \\ ${ }^{2}$ The Centers for Disease Control and Prevention of Xinjiang Uyghur \\ Autonomous Region, Urumqi, China \\ ${ }^{3}$ Department of Ophthalmology, \\ Urumqi General Hospital of Lanzhou Military Command Area, Urumqi, China \\ Corresponding author: H.-Y. Zou \\ E-mail: yijiamin2000@yeah.net
}

Genet. Mol. Res. 13 (1): 1296-1303 (2014)

Received December 18, 2012

Accepted June 7, 2013

Published February 28, 2014

DOI http://dx.doi.org/10.4238/2014.February.28.1

\begin{abstract}
The aim of this study was to investigate the characteristics and polymorphisms of the T-cell receptor BV complementaritydetermining region 3 (TCR $B V C D R 3$ ) gene in peripheral blood mononuclear cells (PBMCs) from patients with uveitis to provide an experimental basis for studying the pathogenesis of this disease. RTPCR amplification of 26 subfamilies of the TCR BV CDR3 gene and immune spectratyping analysis were used to study the pedigree drift of TCR BV CDR3 in PBMCs from the uveitis patients. The following results were obtained: 1) the vast majority of the $T C R B V C D R 3$ spectra in PBMCs in 5 healthy subjects fit the normal (or Gaussian) distribution. The distributions of the TCR BVCDR3 spectra in 4 patients with uveitis were non-normal and showed an abnormal peak including a widowed peak trend, a partial peak, and an irregular abnormal peak. 2) In the 26 TCR BV subfamilies, the abnormal peak frequency was different
\end{abstract}


in the various subfamilies. The BV2 and BV17 (both 3/4) subfamilies had higher frequencies of the non-normally distributed abnormal peak. The BV5.2, BV6, BV15, and BV18 subfamilies showed no abnormal peaks. 3) TCR BV2 and BV17 yielded an abnormal peak in 3 HLAB27-negative patients; however, no such abnormalities were detected in HLA-B27-positive patients. The abnormal expression of some TCR BV subfamilies in PBMCs from patients with uveitis may be associated with the immune pathogenesis of the disease. Our study provides the basis for further investigations into the pathogenesis of uveitis.

Key words: BV subfamily; Complementarity-determining region; Uveitis; T-cell receptor; Immune scanning spectratyping analysis

\section{INTRODUCTION}

Uveitis is a common eye disease. In recent years, it has been the cause of $4-10 \%$ of all cases of blindness and its incidence is rising (Ronday et al., 1994; Suttorp-Schulten and Rothova, 1996). Therefore, uveitis is increasingly attracting the attention of researchers studying blindness. The etiology of the disease is complex, and the majority view is that in addition to trauma, infection, and other factors, autoimmune disorders are the most common and most important cause of uveitis. Therefore, many studies have explored its pathogenesis from an immunological perspective. In 1973, Brewerton et al. first reported that the human leukocyte antigen (HLA) B27 was closely associated with acute anterior uveitis, and additional studies have identified more than 20 HLA-B27-associated antigens of this eye disease (Wakefield et al., 1991).

Examinations of the choroid in uveitis cases have shown that lymphocytes and plasma cells are the main response cells in the intraocular zone of inflammation. The lymphocytes infiltrate the entire choroid with the retina displaying varying degrees of damage (Xiao, 1994), which may reverse if humoral and cellular immune responses are involved in the pathology of uveitis.

In recent years, uveitis has been considered as the result of an antigen-specific T cellmediated autoimmune reaction to a specific eye protein (Whitcup and Nussenblatt, 1997; Dick and Isaacs, 1999). Thus, the involvement of T lymphocytes in the pathogenesis of uveitis has caused widespread concern, and few studies have investigated the role of immune-reactive $T$ cells in uveitis.

The T-cell receptor (TCR) is a specific antigen-recognition molecule on the surface of the T cell, involved in the formation of the TCR germline gene. The allelic exclusion of the TCR $\beta$ chain takes place ahead of the rearrangement of the TCR $\alpha$ chain, which better reflects the characteristics of the T cells in TCR. During the TCR rearrangement process, the complementarity-determining region 3 (CDR3) forms a highly variable region (the NNDNJ region), representing the binding region of the TCR and of the epitopic cluster. Different rearrangements may lead to variable CDR3 lengths, and the characteristics of TCR clonality among different subfamilies can be determined by measuring the length of the CDR3 subfamilies. In this study, the gene scanning technology was used to analyze TCR $B V C D R 3$ pedigree polymorphisms in patients with uveitis. This study provides a basis for exploring the role of $\mathrm{T}$ cells in the mechanisms underlying immune system-induced pathogenesis of uveitis. 


\section{MATERIAL AND METHODS}

\section{Selection of cases}

Four uveitis patients were included in this study, representing both outpatients and hospitalized patients, which were numbered U1-U4. U1-U3 were HLA-B27-negative patients and U4 was an HLA-B27-positive patient with ankylosing spondylitis (AS). The subjects included 1 female (U1) and 3 males (U2 to U4) whose ages ranged from 24-45 years. All subjects were diagnosed with uveitis according to the diagnostic criteria in the literature (Yang, 2009); the diagnostic criteria for the ankylosing spondylitis were those as previously described by van der Linden et al. (1984). Other autoimmune and infectious diseases were excluded. Five healthy subjects were in the control group. Blood $(5 \mathrm{~mL})$ was acquired from all subjects and dipotassium of ethylenediamine tetraacetic acid (EDTA) was added as an anticoagulant.

\section{RNA extraction and cDNA synthesis}

An RNA extraction kit (Omega Bio-Tek, USA) was used to extract total RNA from 2 $\mathrm{x} 10^{6} \mathrm{PBMC}$ from the 4 subjects with uveitis and 2 healthy subjects. The RNA concentration was measured as previously described (Lu and Niu, 2002), and the cDNA was synthesized according to the cDNA Kit conditions.

\section{$T C R B V$ and $T C R B C$ gene primer design and synthesis}

On the basis of previous reports (Zou et al., 2006, 2007), an upstream primer specific for $26 T C R B V$ subfamilies was synthesized. The TCR BC gene shared 1 downstream primer (the terminal end was labeled with the FAM fluorescent marker), and 1 TCR BC control primer was also synthesized. All primers were synthesized by Invitrogen (Shanghai).

\section{PCR amplification of 26 subfamilies of the $T C R B V$ gene}

PCRs were performed in 50- $\mu \mathrm{L}$ volumes containing $2 \mu \mathrm{L}$ cDNA template, $1 \mu \mathrm{L} 10$ $\mathrm{mM}$ dNTP, $5 \mu \mathrm{L}$ 10X PCR buffer (containing $\mathrm{Mg}^{2+}$ ), $2 \mu \mathrm{L}$ TCR BV upstream primer (the concentration of all TCR BV downstream primers was $10 \mu \mathrm{M}), 2 \mu \mathrm{L}$ shared TCR BV-FAM primer, and $1.25 \mathrm{U}$ Taq DNA polymerase. The reaction conditions were as follows: $94^{\circ} \mathrm{C}$ denaturation for $3 \mathrm{~min}$, followed by 35 cycles of $94^{\circ} \mathrm{C}$ for $1 \mathrm{~min}, 55^{\circ} \mathrm{C}$ for $1 \mathrm{~min}$, and $72^{\circ} \mathrm{C}$ for 1 min; a final extension was at $72^{\circ} \mathrm{C}$ extension for $10 \mathrm{~min}$. The PCR products were subjected to electrophoresis on $2 \%$ agarose gels (stained with ethidium bromide), and the remainder were stored at $-20^{\circ} \mathrm{C}$.

\section{GeneScan analysis of T-cell cloning}

Reactions were prepared with $2 \mu \mathrm{L}$ deionized formamide (Hi-Di Formamide, ABI), $0.5 \mu \mathrm{L}$ standard products (ABI Genescan 500-Tamra, which contained different sizes of the luciferase/Tamra-labeled DNA fragments) and $0.5 \mu \mathrm{L}$ sample buffer ( $25 \mathrm{mM}$ EDTA and 50 $\mathrm{mg} / \mathrm{mL}$ Blue Dextran) added to $2 \mu \mathrm{L}$ FAM fluorescence-labeled PCR products from the $T C R$ 
$B V$ gene families. After denaturation at $94^{\circ} \mathrm{C}$ for $4 \mathrm{~min}, 2 \mu \mathrm{L}$ was removed from each tube and loaded onto a $6 \%$ denaturing polyacrylamide gel for electrophoresis for $2 \mathrm{~h}$. The $373 \mathrm{~A}$ DNA Sequence analyzer (ABI, Perkin Elmer Corporation) was used for detection and the GeneScan672 software was used for analysis. Laser scanning during the electrophoresis was used, and the data collected included different colors and intensities of the fluorescein dye, which were recorded as peaks at different positions and of different height and colors.

\section{RESULTS}

\section{RT-PCR results of $T C R B V$ from 26 subfamilies expressed in PBMC}

The TCR $B V$ gene representing 26 subfamilies and the TCR $B C$ gene were expressed in PBMCs from both healthy subjects and patients with uveitis, as indicated by a DNA band of the expected size after $2 \%$ agarose gel electrophoresis (Figure 1).
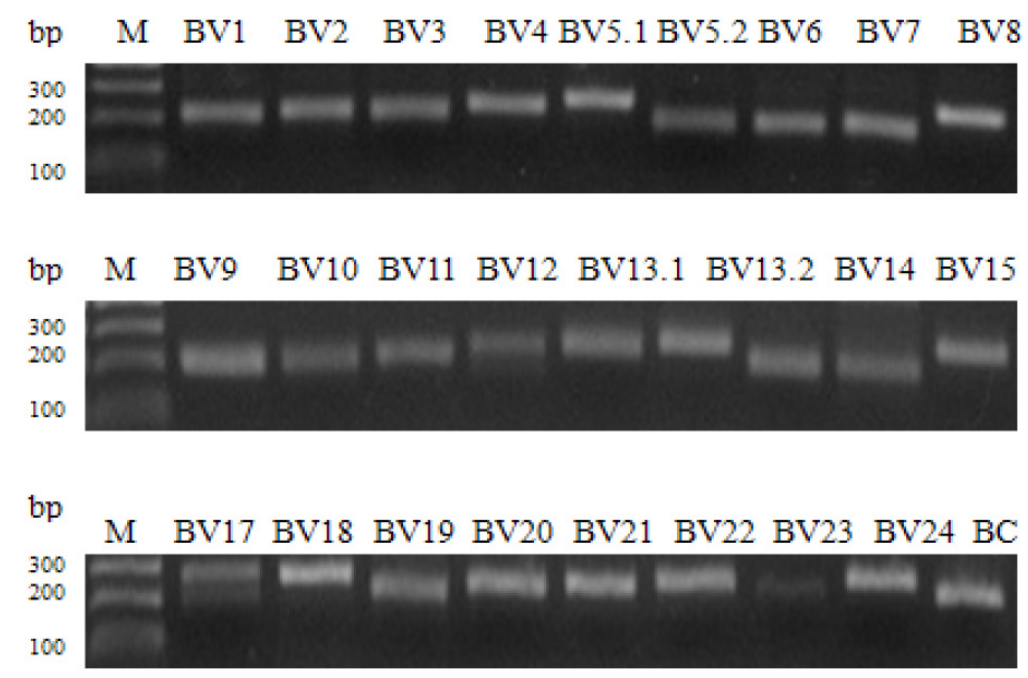

Figure 1. Agarose gel (2\%) eletrophoresis of the PCR products of $T C R B V$ families in a normal control.

\section{$T C R B V$ gene-scan analysis results}

The TCR $B V$ gene representing 26 gene subfamilies expressed in PBMCs from 5 healthy subjects contained 8-10 fragments that differed in size by $3 \mathrm{bp}$. As a result, the spectral map showed 8-10 bell-shaped peaks with a high middle part and low ends. It showed a normal or Gaussian distribution (Figure 2).

Non-normal distributions, indicative of abnormal peaks in the spectral scan of the $T C R B V C D R 3$ subfamilies expressed in peripheral blood, were detected in all uveitis patients. These abnormal peaks included an oligonucleotide peak, a partial peak, and an irregular abnormal peak indicating oligoclonal proliferation of the $\mathrm{T}$ cells or abnormal polyclonal hyperplasia. However, the monoclonal hyperplasia subfamily was not detected in this study. 
The proportion of abnormal peaks of TCR $B V$ from the 26 different subfamilies in the uveitis patients ranged from $31 \%(8 / 26)$ to $50 \%(13 / 26)$, and the $\mathrm{U} 4$ patient displayed the highest rate of abnormal peaks (Table 1). Three to nine oligoclonal trends of hyperplasia were detected in the 26 different $T C R B V$ subfamilies in the uveitis patients, and the $\mathrm{U} 4$ patient (diagnosed with both uveitis and AS) had the highest frequency of the oligoclonal peaks (9/26) (Table 1).

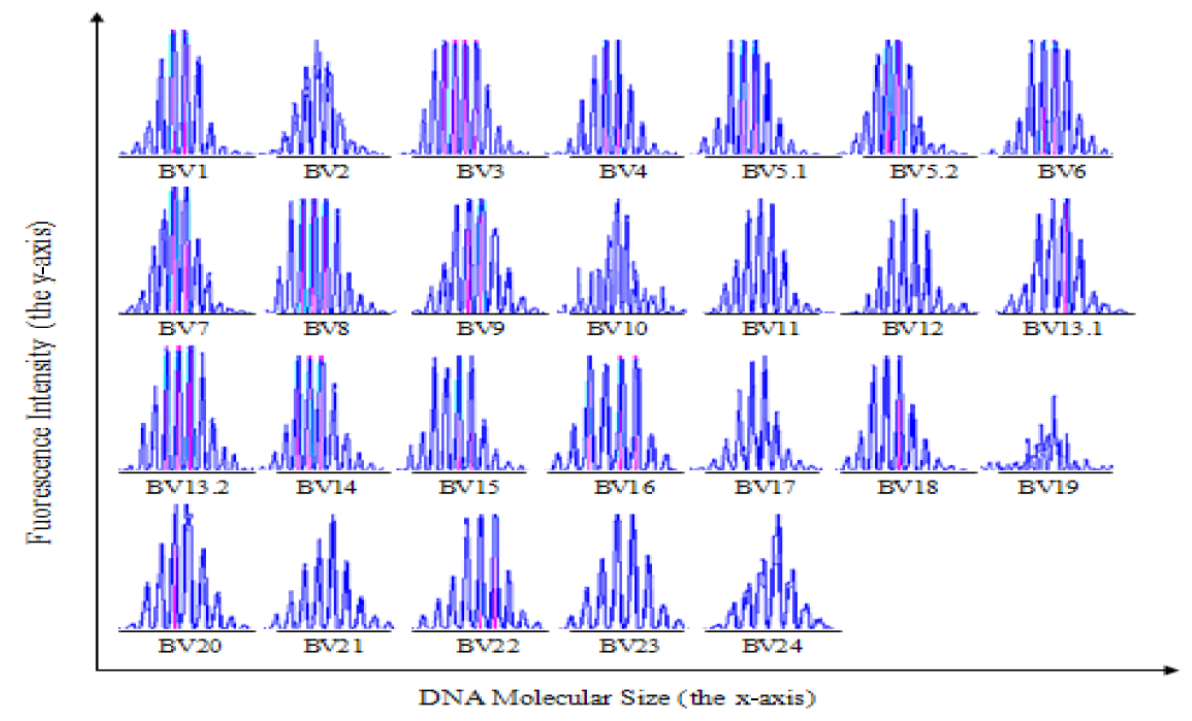

Figure 2. Spectratyping of CDR3 sizes for all $26 T C R B V$ gene families in PBMC in a normal control. The phase difference in the $\mathrm{x}$-axis direction was the size of each RT-PCR product and CDR3 length correspoding to each spectral type. The different height in the $y$-axis direction showed the peak of the relative fluorescence of the corresponding RT-PCR products, which were the expression frequencies of the CDR3.

Table 1. Four cases of uveitis in $T C R B V$ subfamilies with abnormal peak rate.

\begin{tabular}{lllllllc}
\hline Cases & P & A & S & OT & O & M & Abnormal peak rate (\%) \\
\hline 1 & 17 & 4 & 2 & 2 & 1 & 0 & $35(9 / 26)$ \\
2 & 16 & 4 & 2 & 1 & 3 & 0 & $38(10 / 26)$ \\
3 & 18 & 4 & 1 & 1 & 2 & 0 & $31(8 / 26)$ \\
4 & 13 & 3 & 1 & 4 & 5 & 0 & $50(13 / 26)$ \\
\hline
\end{tabular}

$\mathrm{P}=$ polyclonal normal distribution peak; $\mathrm{A}=$ irregular abnormal peak; $\mathrm{S}=$ partial peak; $\mathrm{OT}=$ oligonucleotide peak trend; $\mathrm{O}=$ oligonucleotide peak; $\mathrm{M}=$ single peak.

Among the $26 T C R B V$ gene subfamilies, some subfamilies displayed differences in the frequency of the non-normal abnormal peak. The BV2 and BV17 (both 3/4) subfamilies had higher frequencies of the non-normal abnormal peaks. One the other hand, the BV5.2, BV6, BV15, and BV18 subfamilies exhibited no abnormal peaks. The BV9, BV22, and BV24 (both 2/4) subfamilies showed higher frequencies of the oligonucleotide peak. The BV21 (2/4) subfamily had a higher frequency of the partial peak, and the BV17 (3/4) subfamily had a higher frequency of the irregular abnormal peak. This study also detected abnormal peaks for TCR BV2 and BV17 in the 3 HLA-B27-negative patients (U1-U3); however, no such two abnormalities was detected in the HLA-B27-positive patient (U4) (Figure 3). 


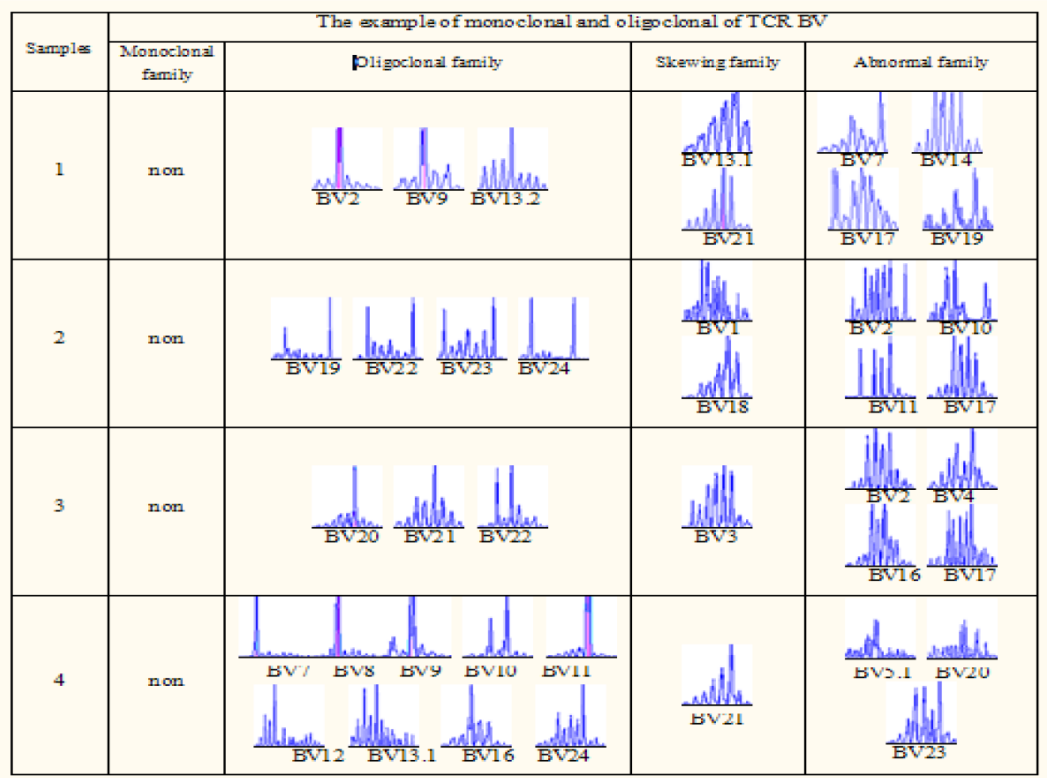

Figure 3. Monoclonal expansion, oligoclonal expansion, skewing family, and abnormal family of $T C R B V$ in the uveitis patients.

\section{DISCUSSION}

Uveitis is caused mainly by autoimmune responses, and a variety of antigens are present in the retina, all of which comprise several active peptides that can induce uveitis (Rai et al., 2001). Yamamoto et al. (1993), stimulating T lymphocytes cultured in vitro with the $\mathrm{S}$ antigen in uveitis patients, observed that the $\mathrm{T}$ cells mounted an immune response. However, this response was not significantly different between the uveitis patients and the normal control group. We speculate that the $\mathrm{S}$ antigen may be a self-concealed antigen. Damage to eye tissues or exposure to other agents triggers an $\mathrm{S}$ antigen-induced immune response that results in uveal inflammatory injury.

Analysis on the clonal characteristics of the TCR BV 26 subfamily genes can help us to understand the nature of the T-cell and immune status. In normal circumstances, when the immune system is unaffected by any antigen stimulation, TCR BV is rearranged randomly, and the $\mathrm{T}$ cells show a positive polyclonal state. However, in case of disease (Long et al., 2006; Matsumoto et al., 2006; Miqueu et al., 2007), stimulation by specific antigens can cause the targeted rearrangement and excessive abnormal cloning of one or a few of the TCR BV subfamilies, and the dominant form of the cloned $\mathrm{T}$ cell may suppress the cloning of other $\mathrm{T}$ cells, which may result in a decrease of immune function.

In this study, the immune scan spectral analysis technology was used to analyze the CDR3 pedigree of the TCR BV subfamilies in healthy subjects, and we observed that the sizes of the RT-PCR products of the 26 subfamilies were variable, indicating that the T cells had undergone diverse rearrangement during germline development. The CDR3 lineage and clonal expression analysis of the TCR $B V$ subfamilies in 4 uveitis patients showed that all of 
them had abnormal spectral changes in the TCR BV subfamilies in peripheral blood T lymphocytes, which suggested that the uveitis patients had abnormal T cells. Some the subfamilies in the uveitis patients displayed expression of oligoclonal hyperplasia that caused multiple changes in the oligoclonal TCR $B V$ subfamilies. Moreover, this also resulted in advantageous hyperplasia of different $T C R B V$ subfamilies in different uveitis patients. A variety of antigens may cause a specific immune response. The TCR BV subfamilies exhibited a pedigree polymorphism and an abnormal clonal proliferation under stimulation with specific antigens. The functional significance of this process and the clonal proliferation of the T-cells in the body is largely unclear and requires further studies. The variety of the different oligoclonal $\mathrm{T}$ cells proliferating in each patient indicated that the uveitis antigen had the polymorphisms. Different patients had different oligoclonal $\mathrm{T}$ cell subfamilies, which may be associated with the different reactivity of each patient.

The results of this study also showed that the U4 patient, who had both AS and uveitis, had the highest amount of oligoclonal proliferation and subfamily abnormality, suggesting that the abnormal expression of TCR BV subfamilies is higher in patients with AS and uveitis than in those who only have uveitis. However, the number of subjects was small in this study, so further research is needed to provide conclusive evidence.

In this study, using spectral scanning analysis of $T C R B V C D R 3$ expression in uveitis patients, we detected only some of the oligoclonal trend hyperplasia subfamilies, and we did not find any of the subfamilies associated with monoclonal hyperplasia.

Further increasing the sample size may provide opportunities for finding additional oligoclonal or even monoclonal hyperplasia subfamilies. This could help to improve our understanding of the characteristics of the TCR BV CDR3 lineage polymorphisms in patients with uveitis. Additional sequence analysis of the oligoclonal hyperplasia CDR3 subfamily may reveal a common, uveitis-associated CDR3 amino acid sequence.

This may clarify the exact role of the uveitis-specific T-cell antigen in immune pathogenesis and provide valuable clues to this uveitis-specific antigen and the immunological pathogenesis, which could open up new ways for targeted immunotherapy.

\section{ACKNOWLEDGMENTS}

Research supported by the National Natural Science Foundation of China (\#81172840).

\section{REFERENCES}

Brewerton DA, Hart FD, Nicholls A, Caffrey M, et al. (1973). Ankylosing spondylitis and HL-A 27. Lancet 1: 904-907.

Dick AD and Isaacs JD (1999). Immunomodulation of autoimmune responses with monoclonal antibodies and immunoadhesins: treatment of ocular inflammatory disease in the next millennium. Br. J. Ophthalmol. 83: 12301234.

Long SA, Khalili J, Ashe J, Berenson R, et al. (2006). Standardized analysis for the quantification of Vbeta CDR3 T-cell receptor diversity. J. Immunol. Meth. 317: 100-113.

Lu XY and Niu JX (2002). Detection of grapevine fan leaf virus by reverse transcription-polymerase chain reaction. Shihezi Univ. 6: 211-213.

Matsumoto Y, Matsuo H, Sakuma H, Park IK, et al. (2006). CDR3 spectratyping analysis of the TCR repertoire in myasthenia gravis. J. Immunol. 176: 5100-5107.

Miqueu P, Guillet M, Degauque N, Dore JC, et al. (2007). Statistical analysis of CDR3 length distributions for the assessment of T and B cell repertoire biases. Mol. Immunol. 44: 1057-1064. 
Rai G, Saxena S, Kumar H and Singh VK (2001). Human retinal S-antigen: T cell epitope mapping in posterior uveitis patients. Exp. Mol. Pathol. 70: 140-145.

Ronday MJ, Stilma JS, Barbe RF, Kijlstra A, et al. (1994). Blindness from uveitis in a hospital population in Sierra Leone. Br. J. Ophthalmol. 78: 690-693.

Suttorp-Schulten MS and Rothova A (1996). The possible impact of uveitis in blindness: a literature survey. $B r . J$. Ophthalmol. 80: 844-848.

van der Linden S, Valkenburg HA and Cats A (1984). Evaluation of diagnostic criteria for ankylosing spondylitis. A proposal for modification of the New York criteria. Arthritis Rheum. 27: 361-368.

Wakefield D, Montanaro A and McCluskey P (1991). Acute anterior uveitis and HLA-B27. Surv. Ophthalmol. 36: 223-232.

Whitcup SM and Nussenblatt RB (1997). Immunologic mechanisms of uveitis. New targets for immunomodulation. Arch. Ophthalmol. 115: 520-525.

Xiao JZ (1994). Therapeutic effect and mechanism of the provincial judge Han tetrandrine on experimental uveitis. Ophthalmology 10: 149-151.

Yamamoto JH, Minami M, Inaba G, Masuda K, et al. (1993). Cellular autoimmunity to retinal specific antigens in patients with Behcet's disease. Br. J. Ophthalmol. 77: 584-589.

Yang PZ (2009). Uveitis Diagnosis and Treatment. People's Medical Publishing House, Beijing, 386-396.

Zou HY, Ma L, Yao XS, Wen Q, et al. (2006). Effects of T cell receptor gene rearrangement on BV CDR3 in Jurkat cells. Nan. Fang Yi. Ke. Da Xue Xue Bao 26: 939-943.

Zou HY, Ma L, Meng MJ, Yao XS, et al. (2007). Expression of recombination-activating genes and T cell receptor gene recombination in the human T cell leukemia cell line. Chin. Med. J. 120: 410-415. 